\title{
Semidiscretization for a Doubly Nonlinear Parabolic Equation Related to the $\mathbf{p}(\mathbf{x})$-Laplacian
}

\author{
Hamid El Bahja, Abderrahmane El Hachimi $\mathbb{D}^{D}$, and Ali Alami Idrissi \\ Center of Mathematical Research and Applications of Rabat (CeReMAR), Laboratory of Mathematical Analysis and Applications \\ (LAMA), Department of Mathematics, Faculty of Sciences, Mohammed V University, P.O. Box 1014, Rabat, Morocco
}

Correspondence should be addressed to Abderrahmane El Hachimi; aelhachi@yahoo.fr

Received 28 January 2019; Accepted 31 March 2019; Published 11 April 2019

Guest Editor: Shapour Heidarkhani

Copyright (C) 2019 Hamid El Bahja et al. This is an open access article distributed under the Creative Commons Attribution License, which permits unrestricted use, distribution, and reproduction in any medium, provided the original work is properly cited.

This paper studies a time discretization for a doubly nonlinear parabolic equation related to the $\mathrm{p}(\mathrm{x})$-Laplacian by using Eulerforward scheme. We investigate existence, uniqueness, and stability questions and prove existence of the global compact attractor.

\section{Introduction}

The investigation of the asymptotic behavior for nonlinear parabolic equations involving the so-called p-Laplacian operator has been addressed by several authors in the last decades, in both bounded and unbounded domains, with constant or variable exponents (see [1-8]). One way to treat this question is to analyze the existence and structure (regularity and finite or infinite dimensionality of the attractor generated by the solutions of the governed equation (see [9]). The existence of the global attractor for the related semigroup acting on the natural weak energy space $L^{p}(\Omega)$ has been proved in $[7,10,11]$.

In this paper, our goal is to study the time discretization for a doubly nonlinear parabolic equation associated with the $\mathrm{p}(\mathrm{x})$-Laplacian, where in addition to usual questions of existence, uniqueness, and stability of the solutions, we will be concerned with the existence of absorbing sets and the global attractor as well. The problem under consideration is of the form

$$
\begin{aligned}
\frac{\partial \beta(u)}{\partial t}-\Delta_{p(x)} u+f(x, t, u) & =0 \quad \text { in } \Omega \times] 0, \infty[, \\
u & =0 \quad \text { on } \partial \Omega \times] 0, \infty[, \\
\left.\beta(u)\right|_{t=0} & =\beta\left(u_{0}\right) \quad \text { in } \Omega .
\end{aligned}
$$

where $\Delta_{p(x)} u=\operatorname{div}\left(|\nabla u|^{p(x)-2} \nabla u\right), p \in C(\bar{\Omega})$ with $1<$ $p(x)<+\infty, \beta$ is a nonlinearity of porous media type, $f$ is a nonlinearity of reaction type, and $\Omega$ is an open bounded set of $\mathbb{R}^{N}$ with smooth boundary.

Existence results and qualitative properties concerning the solutions of the continuous problem (1) and more general problems have been obtained by many authors in the last decade. We cote the papers $[1-6,12]$ and the references therein.

Our motivation to study problem (1) is the fact that it is considered in particular as a model of an important class of non-Newtonian fluids which are well known as electrorheological fluids (see [13]).

This paper is organized as follows: In Section 2, we give some preliminaries and notation. In Section 3, we discretize problem (1) by using Euler-forward scheme and obtain existence, uniqueness, and stability results.

Finally, in Section 4 we show the existence of absorbing sets in $W_{0}^{1, p(x)}(\Omega) \cap L^{\infty}(\Omega)$, which in turn ensures the existence of a compact global attractor.

\section{Preliminaries}

We begin with a review of some basic results that will be needed in the subsequent sections. The known results are stated without proofs. We shall however provide references where the proofs can be found.

We first introduce the space $L^{p(.)}(\Omega)$ and $W^{1, p(.)}(\Omega)$ and state some of their properties. 
Let $\Omega$ stand for a regular open bounded set of $\mathbb{R}^{N}$ and $p: \Omega \longrightarrow] 1,+\infty[$ be a measurable bounded function as a variable exponent. Denote

$$
\begin{aligned}
p^{-} & :=\operatorname{ess} \inf _{x \in \Omega} p(x) \\
\text { and } p^{+} & :=\sup \sin _{x \in \Omega} p(x) .
\end{aligned}
$$

We define the variable exponent Lebesgue space $L^{p(x)}(\Omega)$ by

$$
L^{p(x)}(\Omega)=\{u ;
$$

$u$ is a mesurable real-valued function, $\rho_{p(x)}(u)$

$$
\left.=\int_{\Omega}|u(x)|^{p(x)} d x<\infty\right\},
$$

endowed with the Luxembourg norm

$$
\|u\|_{p(x)}=\inf \left\{\lambda>0, \int_{\Omega}\left|\frac{u(x)}{\lambda}\right|^{p(x)} \leq 1\right\} .
$$

The following results can be found in [14-17].

Lemma 1. Let $p: \Omega \longrightarrow] 1,+\infty[$ be a measurable function with $1<p^{-} \leq p(x) \leq p^{+}<\infty$. Then, we have

$$
\begin{aligned}
& \min \left\{\rho_{p(x)}(u)^{1 / p^{-}}, \rho_{p(x)}(u)^{1 / p^{+}}\right\} \leq\|u\|_{p(x)} \\
& \leq \max \left\{\rho_{p(x)}(u)^{1 / p^{-}}, \rho_{p(x)}(u)^{1 / p^{+}}\right\},
\end{aligned}
$$

for all $u \in L^{p(x)}(\Omega)$.

Proposition 2. The space $\left(L^{p(x)}(\Omega),\|\cdot\|_{p(x)}\right)$ is a separable, uniform convex Banach space, and its conjugate space is $L^{q(x)}(\Omega)$, where $1 / p(x)+1 / q(x)=1$. Moreover, for any $u \in$ $L^{p(x)}(\Omega)$ and $v \in L^{q(x)}(\Omega)$, we have

$$
\left|\int_{\Omega} u v d x\right| \leq\left(\frac{1}{p^{-}}+\frac{1}{q^{-}}\right)\|u\|_{p(x)}\|v\|_{q(x)} .
$$

Let $W^{1, p(x)}(\Omega)$ denote the space of measurable functions $u$ such that $u$ and the distributional derivative $\nabla u$ are in $L^{p(x)}(\Omega)$. The norm

$$
\|u\|_{1, p(x)}=\|u\|_{p(x)}+\|\nabla u\|_{p(x)}
$$

makes $W^{1, p(x)}(\Omega)$ a Banach space.

Let

$$
\begin{aligned}
& C_{+}(\bar{\Omega})=\left\{p(x): \bar{\Omega} \longrightarrow\left[p^{-}, p^{+}\right]\right. \\
& \quad C(1, \infty) ; p \text { is a continous function }\},
\end{aligned}
$$

We say that $p \in C_{+}(\Omega)$ satisfies the log-Hölder condition in $\Omega$ if

$$
\begin{gathered}
\forall x, y \in \Omega, \\
|x-y|<1, \\
|p(x)-p(y)| \leq \omega(|x-y|),
\end{gathered}
$$

where $\omega$ satisfies

$$
\limsup _{\tau \rightarrow 0^{+}} \omega(\tau) \ln \left(\frac{1}{\tau}\right)<\infty .
$$

It is well known that if $p(x)$ satisfies the log-Hölder condition (9), then the space $C^{\infty}(\Omega)$ is dense in $W^{1, p(x)}(\Omega)$. Moreover, we can define the Sobolev space with zero boundary values, $W_{0}^{1, p(x)}(\Omega)$ as the completion of $C_{0}^{\infty}(\Omega)$, with respect to the norm $\|\cdot\|_{W^{1, p(x)}(\Omega)}$.

Let us recall the following versions of Poincarés inequality.

Lemma 3. If $p: \Omega \longrightarrow] 1,+\infty[$ is continuous in $\bar{\Omega}$, then there exists a constant $C$ such that

$$
\|u\|_{p(x)} \leq C\|\nabla u\|_{p(x)},
$$

for all $u \in W_{0}^{1, p(x)}(\Omega)$ and thus $\|u\|_{1, p(x)}$ and $\|\nabla u\|_{p(x)}$ are equivalent norms in $W_{0}^{1, p(x)}(\Omega)$.

Let us next consider the modular version of Poincaré's inequality.

Lemma 4. Let $p(x)$ be an element of $L^{\infty}(\Omega)$ and let $u \in$ $W^{1, p(x)}(\Omega)$. There exists a constant $C$ depending only on $\Omega$ such that

$$
\rho_{p(x)}(u) \leq C \rho_{p(x)}(\nabla u) .
$$

\section{The Semidiscretized Problem: Existence, Uniqueness, and Stability}

Let $\beta$ be a continuous increasing function with $\beta(0)=0$. For $t \in \mathbb{R}$, we set

$$
\psi(t)=\int_{0}^{t} \beta(\tau) d \tau .
$$

We consider the following Euler-forward scheme associated with (1):

$$
\begin{aligned}
\beta\left(U^{n}\right)-\tau \Delta_{p(x)} U^{n}+\tau f\left(x, n \tau, U^{n}\right) & =\beta\left(U^{n-1}\right) \\
\text { in } \Omega, & \\
U^{n} & =0 \quad \text { on } \partial \Omega, \\
\beta\left(U^{0}\right) & =\beta\left(u_{0}\right) \quad \text { in } \Omega,
\end{aligned}
$$

where $N \tau=T$, with $T$ being a fixed positive real, and $1 \leq$ $n \leq N$. We shall be concerned with the following two cases: $u_{0} \in L^{\infty}(\Omega)$ or $u_{0} \in L^{2}(\Omega)$.

3.1. Case 1: $u_{0} \in L^{\infty}(\Omega)$. We assume the following hypotheses:

$\left(H_{1}\right)$ the function $\beta$ is an increasing and continuous from $\mathbb{R}$ to $\mathbb{R}$ such that $\beta(u) \leq C|u|^{\alpha-1}$ for any $u \in \mathbb{R}$ with $1 \leq \alpha<$ $p^{-}$. 
$\left(H_{2}\right)$ for $\xi \in \mathbb{R}$, the map $(x, t) \longmapsto f(x, t, \xi)$ is measurable and, a.e. in $\Omega \times \mathbb{R}^{+}, \xi \longmapsto f(x, t, \xi)$ is continuous. Furthermore, we assume that there exists $C_{1}>0$, such that, for a.e. $(x, t) \in \Omega \times \mathbb{R}^{+}$, we have $\operatorname{sign}(\xi) . f(x, t, \xi) \geq-C_{1}$.

$\left(H_{3}\right)$ there exists $C_{2}>0$, such that, for almost $(x, t) \in$ $\Omega \times \mathbb{R}^{+}, \xi \longmapsto f(x, t, \xi)+C_{2} \beta(\xi)$ is increasing.

Lemma 5. Assume $\left(H_{1}\right)$ and $\left(H_{2}\right)$. Then, for all $n \in\{0, . ., N\}$, we have $U^{n} \in L^{\infty}(\Omega)$.

Proof. To show that $U^{1} \in L^{\infty}(\Omega)$, we can write (17) as

$$
\begin{aligned}
-\tau \Delta_{p(x)} U^{1} & =\beta\left(u_{0}\right)-\beta\left(U^{1}\right)-\tau f\left(x, \tau, U^{1}\right), \\
U^{1} & \in W_{0}^{1, p(x)} .
\end{aligned}
$$

Then, by $\left(H_{1}\right),\left(H_{2}\right)$, and Theorem 4.1 of [18], we can conclude that $U^{1} \in L^{\infty}(\Omega)$. Then, by a simple induction, we deduce that $U^{n} \in L^{\infty}(\Omega)$ for all $n=0, . ., N$.

Theorem 6. Assume $\left(H_{1}\right),\left(H_{2}\right)$, and $\left(H_{3}\right)$. For $n=0, \ldots, N$, there exists a unique solution $U^{n}$ of $(14)$ in $W_{0}^{1, p(x)}(\Omega) \cap L^{\infty}(\Omega)$ provided that $0<\tau<1 / C_{2}$.

Proof. We can write (14) as

$$
\begin{aligned}
-\tau \Delta_{p(x)} U^{n} & =\beta\left(U^{n-1}\right)-\beta\left(U^{n}\right)-\tau f\left(x, n \tau, U^{n}\right) \\
U^{n} & \in W_{0}^{1, p(x)}(\Omega) .
\end{aligned}
$$

By using $\left(H_{1}\right),\left(H_{2}\right)$, and applying Theorem 4.3 of [19] and Lemma 5 , we deduce the existence of at least one solution $U^{n} \in W_{0}^{1, p(x)}(\Omega) \cap L^{\infty}(\Omega)$ for $n=1, \ldots, N$.

Let us now prove the uniqueness. For simplicity, we set

$$
\begin{aligned}
\omega & =U^{n}, \\
\bar{f}(x, \omega) & =f\left(x, n \tau, U^{n}\right), \\
\text { and } g(x) & =\beta\left(U^{n-1}\right) .
\end{aligned}
$$

Then, problem (14) reads

$$
-\tau \Delta_{p(x)} \omega+\tau \bar{f}(x, \omega)+\beta(\omega)=g(x), \quad \omega \in W_{0}^{1, p(x)} .
$$

If $\omega_{1}$ and $\omega_{2}$ are two solutions of (14), then

$$
\begin{aligned}
& -\tau \Delta_{p(x)} \omega_{1}+\tau \Delta_{p(x)} \omega_{2}+\tau\left(\bar{f}\left(x, \omega_{1}\right)-\bar{f}\left(x, \omega_{2}\right)\right) \\
& +\beta\left(\omega_{1}\right)-\beta\left(\omega_{2}\right)=0
\end{aligned}
$$

Multiplying (19) by $\omega_{1}-\omega_{2}$ and integrating over $\Omega$ give

$$
\begin{aligned}
& \left\langle-\tau \Delta_{p(x)} \omega_{1}+\tau \Delta_{p(x)} \omega_{2}, \omega_{1}-\omega_{2}\right\rangle \\
& \quad+\tau \int_{\Omega}\left(\bar{f}\left(x, \omega_{1}\right)-\bar{f}\left(x, \omega_{2}\right)\right)\left(\omega_{1}-\omega_{2}\right) d x \\
& \quad+\int_{\Omega}\left(\beta\left(\omega_{1}\right)-\beta\left(\omega_{2}\right)\right)\left(\omega_{1}-\omega_{2}\right) d x=0,
\end{aligned}
$$

where $\langle.,$.$\rangle denotes the pairing between W^{1, p(x)}(\Omega)$ and $W^{-1, q(x)}(\Omega)$.

Then, applying $\left(H_{3}\right)$ yields

$$
\begin{aligned}
& \int_{\Omega}\left(\bar{f}\left(x, \omega_{1}\right)-\bar{f}\left(x, \omega_{2}\right)\right)\left(\omega_{1}-\omega_{2}\right) d x \\
& \quad \geq-C_{2} \int_{\Omega}\left(\beta\left(\omega_{1}\right)-\beta\left(\omega_{2}\right)\right)\left(\omega_{1}-\omega_{2}\right) d x .
\end{aligned}
$$

Now by using (21) and the monotonicity of the $\mathrm{p}(\mathrm{x})$-Laplacian operator, (20) reduces to

$$
\left(1-\tau C_{2}\right) \int_{\Omega}\left(\beta\left(\omega_{1}\right)-\beta\left(\omega_{2}\right)\right)\left(\omega_{1}-\omega_{2}\right) d x \leq 0
$$

Then by $\left(H_{1}\right)$, we get $\omega_{1}=\omega_{2}$ for $\tau<1 / C_{2}$.

Theorem 7. Assume $\left(H_{1}\right)$ and $\left(H_{2}\right)$. Then, there exists a constant $C\left(T, u_{0}\right)>0$, depending on $T, u_{0}, \beta$, and $\Omega$, but not on $N$, such that, for all $n=1, . ., N$,

(i)

$$
\left\|U^{n}\right\|_{\infty} \leq C\left(T, u_{0}\right)
$$

(ii)

$$
\int_{\Omega} \psi^{*}\left(\beta\left(U^{n}\right)\right) d x+\tau \sum_{k=1}^{n}\left\|U^{k}\right\|_{1, p(x)}^{\alpha} \leq C\left(T, u_{0}\right),
$$

where $\alpha$ depends either on $p^{-}$or $p^{+}$,

(iii)

$$
\sum_{k=1}^{n}\left\|\beta\left(U^{k}\right)-\beta\left(U^{k-1}\right)\right\|_{2}^{2} \leq C\left(T, u_{0}\right) .
$$

Proof. (i) From Lemma 5, we have $U^{n} \in L^{\infty}(\Omega)$. Then, multiplying (14) by $\left|\beta\left(U^{n}\right)\right|^{k} \beta\left(U^{n}\right)$ and integrating over $\Omega$, we get

$$
\begin{aligned}
& \int_{\Omega}\left|\beta\left(U^{n}\right)\right|^{k+2} d x-\tau \int_{\Omega} \Delta_{p(x)} U^{n}\left|\beta\left(U^{n}\right)\right|^{k} \beta\left(U^{n}\right) d x \\
& \quad+\tau \int_{\Omega}\left|\beta\left(U^{n}\right)\right|^{k} \beta\left(U^{n}\right) f\left(x, n \tau, U^{n}\right) d x \\
& =\int_{\Omega}\left|\beta\left(U^{n}\right)\right|^{k} \beta\left(U^{n}\right) \beta\left(U^{n-1}\right) d x .
\end{aligned}
$$

Since $\beta(0)=0$ and $\beta$ and $-\Delta_{p(x)}$ are monotone, then we have

$$
-\tau \int_{\Omega} \Delta_{p(x)} U^{n}\left|\beta\left(U^{n}\right)\right|^{k} \beta\left(U^{n}\right) d x \geq 0 .
$$

Therefore, we obtain

$$
\begin{aligned}
\left\|\beta\left(U^{n}\right)\right\|_{k+2}^{k+2} \leq & \left\|\beta\left(U^{n}\right)\right\|_{k+2}^{k+1}\left\|\beta\left(U^{n-1}\right)\right\|_{k+2} \\
& +C \tau\left\|\beta\left(U^{n}\right)\right\|_{k+2}^{k+1} .
\end{aligned}
$$

Hence,

$$
\left\|\beta\left(U^{n}\right)\right\|_{k+2} \leq\left\|\beta\left(U^{n-1}\right)\right\|_{k+2}+C \tau\left\|\beta\left(U^{n}\right)\right\|_{k+2}^{k+1} .
$$


By simple induction, we get

$$
\left\|\beta\left(U^{n}\right)\right\|_{k+2} \leq\left\|\beta\left(U_{0}\right)\right\|_{k+2}+N C \tau .
$$

Finally, as $k \longrightarrow \infty$, we obtain (23).

(ii) In order to prove (24), we multiply (14) by $U^{k}$ (with $\mathrm{k}$ instead of $\mathrm{n})$. By using $\left(\mathrm{H}_{2}\right)$, we get

$$
\begin{aligned}
& \int_{\Omega}\left(\beta\left(U^{k}\right)-\beta\left(U^{k-1}\right)\right) U^{k} d x+\tau \rho_{p(x)}\left(\nabla U^{k}\right) \\
& \quad \leq \tau C_{1}\left\|U^{k}\right\|_{1} .
\end{aligned}
$$

Thanks to the properties of the Legendre transformation, we get

$$
\begin{gathered}
\int_{\Omega} \psi^{*}\left(\beta\left(U^{k}\right)\right) d x-\int_{\Omega} \psi^{*}\left(\beta\left(U^{k-1}\right)\right) d x \\
\leq \int_{\Omega}\left(\beta\left(U^{k}\right)-\beta\left(U^{k-1}\right)\right) U^{k} d x .
\end{gathered}
$$

Then, we have

$$
\begin{gathered}
\int_{\Omega} \psi^{*}\left(\beta\left(U^{k}\right)\right) d x-\int_{\Omega} \psi^{*}\left(\beta\left(U^{k-1}\right)\right) d x \\
+\tau \rho_{p(x)}\left(\nabla U^{k}\right) \leq \tau C_{1}\left\|U^{k}\right\|_{L^{1}(\Omega)} .
\end{gathered}
$$

Finally, after summation of (33) from $k=1$ to $n$, we deduce that

$$
\begin{aligned}
& \int_{\Omega} \psi^{*}\left(\beta\left(U^{n}\right)\right) d x \\
& \quad+\tau \sum_{k=1}^{n} \min \left(\left\|U^{k}\right\|_{1, p(x)}^{p^{+}},\left\|U^{k}\right\|_{1, p(x)}^{p^{-}}\right) \\
& \leq \tau C_{1} \sum_{k=1}^{n}\left\|U^{k}\right\|_{L^{1}(\Omega)}+\int_{\Omega} \psi^{*}\left(\beta\left(u_{0}\right)\right) d x .
\end{aligned}
$$

We set

$$
\left\|U^{k}\right\|_{p(x)}^{\alpha}=\min \left(\left\|U^{k}\right\|_{1, p(x)}^{p^{+}},\left\|U^{k}\right\|_{1, p(x)}^{p^{-}}\right) .
$$

Then, the continuity of $\beta$ and the use of Lemma 5 allow us to conclude to the proof of point (24).

(iii) To prove point (25), we multiply the first equation of (17) by $\beta\left(U^{k}\right)$. By using $\left(H_{2}\right)$, we get

$$
\begin{aligned}
& \int_{\Omega}\left(\beta\left(U^{k}\right)-\beta\left(U^{k-1}\right)\right) \beta\left(U^{k}\right) d x \\
& \quad-\tau \int_{\Omega} \Delta_{p(x)} U^{k} \beta\left(U^{k}\right) d x \leq C_{1} \tau\left\|\beta\left(U^{k}\right)\right\|_{L^{1}(\Omega)} .
\end{aligned}
$$

With the aid of the elementary identity,

$$
2 a(a-b)=a^{2}-b^{2}+(a-b)^{2},
$$

for any reals $a$ and $b$, we get from (36) that

$$
\begin{aligned}
& \left\|\beta\left(U^{k}\right)\right\|_{L^{2}(\Omega)}^{2}-\left\|\beta\left(U^{k-1}\right)\right\|_{L^{2}(\Omega)}^{2} \\
& \quad+\left\|\beta\left(U^{k}\right)-\beta\left(U^{k-1}\right)\right\|_{L^{2}(\Omega)}^{2} \\
& \leq C \tau\left\|\beta\left(U^{k}\right)\right\|_{L^{1}(\Omega)} .
\end{aligned}
$$

Now, we take the sum of (38) from $k=1$ to $n$ to obtain

$$
\begin{aligned}
& \left\|\beta\left(U^{n}\right)\right\|_{L^{2}(\Omega)}^{2}+\sum_{k=1}^{n}\left\|\beta\left(U^{k}\right)-\beta\left(U^{k-1}\right)\right\|_{L^{2}(\Omega)}^{2} \\
& \leq C \tau\left\|\beta\left(U^{k}\right)\right\|_{L^{1}(\Omega)}+\left\|\beta\left(u_{0}\right)\right\|_{L^{2}(\Omega)}^{2} .
\end{aligned}
$$

Thus, by $\left(H_{1}\right)$ and Lemma 5 we deduce (25).

Lemma 8. For all $u, v \in W_{0}^{1, p(x)}(\Omega)$, there exists a positive constant $\alpha$ depending either on $p^{+}$or $p^{-}$such that, for $1<$ $p(x)<2$, we have

$$
\rho_{p(x)}(\nabla(u-v))^{2 / \alpha} \leq C\left\langle-\Delta_{p(x)} u+\Delta_{p(x)} v, u-v\right\rangle .
$$

Proof. If $1<p(x)<2$, for any $x \in \Omega$, then we have the following inequality for any $\xi, \eta \in \mathbb{R}^{N}$ :

$$
\begin{aligned}
& (|\xi|+|\eta|)^{2-p(x)}\left(|\xi|^{p(x)-2} \xi-|\eta|^{p(x)-2} \eta\right)(\xi-\eta) \\
& \quad \geq \delta|\xi-\eta|^{2}
\end{aligned}
$$

By setting $\xi=\nabla u$ and $\eta=\nabla v$ and integrating over $\Omega$, we get

$$
\begin{aligned}
& \delta^{p^{+} / 2} \int_{\Omega}|\nabla u-\nabla v|^{p(x)} d x \\
& \quad \leq \int_{\Omega}\left(\left(|\nabla u|^{p(x)-2} \nabla u-|\nabla v|^{p(x)-2} \nabla v\right) .\right. \\
& \quad \nabla(u-v))^{p(x) / 2} \times(|\nabla u|+|\nabla v|)^{(2-p(x)) p(x) / 2} d x .
\end{aligned}
$$

Then, by Holder's inequality we get

$$
\begin{aligned}
& \delta^{p^{+} / 2} \int_{\Omega}|\nabla u-\nabla v|^{p(x)} d x \\
& \leq \|\left(\left(|\nabla u|^{p(x)-2} \nabla u-|\nabla v|^{p(x)-2} \nabla v\right) .\right. \\
& \quad \nabla(u-v))^{p(x) / 2}\left\|_{2 / p(x)} \times\right\|(|\nabla u| \\
& +|\nabla v|)^{(2-p(x)) p(x) / 2} \|_{2 /(2-p(x))} .
\end{aligned}
$$

Let

$$
\begin{aligned}
& I=\int_{\Omega}\left(|\nabla u|^{p(x)-2} \nabla u-|\nabla v|^{p(x)-2} \nabla v\right) \cdot \nabla(u-v) d x \\
& \text { and } J=\int_{\Omega}(|\nabla u|+|\nabla v|)^{p(x)} d x,
\end{aligned}
$$

and $\alpha$ and $\xi$ be such that

$$
(I)^{\alpha / 2}=\max \left((I)^{p^{-} / 2},(I)^{p^{+} / 2}\right)
$$

and

$$
J^{\xi}=\max \left((J)^{\left(2-p^{-}\right) / 2},(J)^{\left(2-p^{+}\right) / 2}\right)
$$


Then, we get

$$
\delta^{p^{+} / 2} \int_{\Omega}|\nabla u-\nabla v|^{p(x)} d x \leq 2^{\xi p^{+}}(I)^{\alpha / 2} J^{\xi}
$$

Therefore, we have

$$
\begin{aligned}
& \delta^{p^{+} / 2} \int_{\Omega}|\nabla u-\nabla v|^{p(x)} d x \\
& \leq 2^{\xi p^{+}}\left\langle-\Delta_{p(x)} u+\Delta_{p(x)} v, u-v\right\rangle^{\alpha / 2} \\
& \cdot\left(\rho_{p(x)}(\nabla u)+\rho_{p(x)}(\nabla v)\right)^{\xi} .
\end{aligned}
$$

Hence, by (24) of Theorem 7 we get the desired result.

Lemma 9. Assume $p(x) \geq 2$. Then, for all $u, v \in W_{0}^{1, p(x)}(\Omega)$, we have

$$
\begin{aligned}
& \left(\frac{1}{2}\right)^{p^{-}} \rho_{p(x)}(\nabla(u-v)) \\
& \quad \leq\left\langle-\Delta_{p(x)} u+\Delta_{p(x)} v, u-v\right\rangle .
\end{aligned}
$$

Proof. As $p(x) \geq 2$, for any $x \in \Omega$, then we have the following inequality for any $\xi, \eta \in \mathbb{R}^{N}$ :

$$
\left(|\xi|^{p(x)-2} \xi-|\eta|^{p(x)-2} \eta\right)(\xi-\eta) \geq\left(\frac{1}{2}\right)^{p-}|\xi-\eta|^{p(x)}
$$

By setting $\xi=\nabla u$ and $\eta=\nabla v$ and integrating over $\Omega$, we get

$$
\begin{aligned}
& \left(\frac{1}{2}\right)^{p-} \int_{\Omega}|\nabla u-\nabla v|^{p(x)} d x \\
& \quad \leq \int_{\Omega}\left(|\nabla u|^{p(x)-2} \nabla u-|\nabla v|^{p(x)-2} \nabla v\right)(\nabla(u-v)) d x
\end{aligned}
$$

Hence

$$
\left(\frac{1}{2}\right)^{p^{-}} \rho_{p(x)}(\nabla(u-v)) \leq\left\langle-\Delta_{p(x)} u+\Delta_{p(x)} v, u-v\right\rangle .
$$

We can also derive a uniqueness result for problem (17) if we replace $\left(\mathrm{H}_{3}\right)$ by the following hypothesis:

$\left(H_{4}\right)$ for all $M>0$, there exists $C_{M}>0$ such that, if $|\xi|+$ $\left|\xi^{\prime}\right| \leq M$, then

$$
\begin{aligned}
& \left|f(t, x, \xi)-f\left(t, x, \xi^{\prime}\right)\right|^{\theta} \\
& \quad \leq C_{M}\left(\beta(\xi)-\beta\left(\xi^{\prime}\right)\right)\left(\xi-\xi^{\prime}\right),
\end{aligned}
$$

where

$$
\theta=\left\{\begin{array}{ll}
\sigma^{\prime} & \text { for } 1<p(x)<2, \\
p^{\prime-} & \text { for } p(x) \geq 2,
\end{array} \quad \text { for all } x \in \Omega,\right.
$$

with $\sigma^{\prime}$ being a positive constant to be prescribed below.
Proposition 10. Assume $\left(H_{1}\right),\left(H_{2}\right)$, and $\left(H_{4}\right)$. Then, problem (14) has a unique solution for all $0<\tau<\eta$, where $\eta$ is a prescribed constant.

Proof. Let $\omega_{1}$ and $\omega_{2}$ be two solutions of (14).

First case: suppose that $1<p(x)<2$, for all $x \in \Omega$. Then, from (20) and by using Lemma 8 and Holder's inequality, we get

$$
\begin{aligned}
& \tau C \rho_{p(x)}\left(\nabla\left(\omega_{1}-\omega_{2}\right)\right)^{2 / \alpha} \\
& \quad+\int_{\Omega}\left(\beta\left(\omega_{1}\right)-\beta\left(\omega_{2}\right)\right)\left(\omega_{1}-\omega_{2}\right) d x \\
& \leq \tau\left\|\bar{f}\left(x, \omega_{1}\right)-\bar{f}\left(x, \omega_{2}\right)\right\|_{L^{\infty}(\Omega)}\left\|\omega_{1}-\omega_{2}\right\|_{L^{1}(\Omega)} .
\end{aligned}
$$

Let $\lambda$ be such that

$$
\begin{aligned}
& \rho_{p(x)}\left(\omega_{1}-\omega_{2}\right)^{1 / \lambda} \\
& =\max \left(\rho_{p(x)}\left(\omega_{1}-\omega_{2}\right)^{1 / p^{-}}, \rho_{p(x)}\left(\omega_{1}-\omega_{2}\right)^{1 / p^{+}}\right),
\end{aligned}
$$

and

$$
\begin{aligned}
\sigma & =\frac{2 \lambda}{\alpha}, \\
\frac{1}{\sigma}+\frac{1}{\sigma^{\prime}} & =1 .
\end{aligned}
$$

Then, by $\left(H_{1}\right),\left(H_{2}\right)$, and $\left(H_{4}\right)$, Lemma 4 , and Young's inequality, we get

$$
\begin{aligned}
& \tau C \rho_{p(x)}\left(\nabla\left(\omega_{1}-\omega_{2}\right)\right)^{2 / \alpha} \\
& \leq\left(\frac{1}{\sigma^{\prime}}-1\right) \int_{\Omega}\left(\beta\left(\omega_{1}\right)-\beta\left(\omega_{2}\right)\right)\left(\omega_{1}-\omega_{2}\right) d x \\
& \quad+C^{\prime} \tau^{\sigma} \rho_{p(x)}\left(\nabla\left(\omega_{1}-\omega_{2}\right)\right)^{2 / \alpha} .
\end{aligned}
$$

Therefore, for $0<\tau<\left(C / C^{\prime}\right)^{1 /(\sigma-1)}$, we get $\omega_{1}=\omega_{2}$.

Second case: suppose that $p(x) \geq 2$, for all $x \in \Omega$. From (20) and by using Lemma 9 and Young's inequality, we get

$$
\begin{aligned}
& \tau\left(\frac{1}{2}\right)^{p^{-}} \rho_{p(x)}\left(\nabla\left(\omega_{1}-\omega_{2}\right)\right) \\
& \quad+\int_{\Omega}\left(\beta\left(\omega_{1}\right)-\beta\left(\omega_{2}\right)\right)\left(\omega_{1}-\omega_{2}\right) d x \\
& \leq \frac{1}{p^{\prime-} C_{M}} \int_{\Omega}\left|\left(\bar{f}\left(x, \omega_{1}\right)-\bar{f}\left(x, \omega_{2}\right)\right)\right|^{p^{\prime}(x)} d x \\
& \quad+\frac{\tau^{p^{+} C_{M}^{p^{+} / p^{\prime-}}}}{p^{-}} \int_{\Omega}\left|\omega_{1}-\omega_{2}\right|^{p(x)} d x .
\end{aligned}
$$


Then, by using $\left(H_{1}\right)$ and $\left(H_{4}\right)$ we get

$$
\begin{aligned}
& \tau\left(\frac{1}{2}\right)^{p^{-}} \rho_{p(x)}\left(\nabla\left(\omega_{1}-\omega_{2}\right)\right) \\
& \leq\left(\frac{1}{p^{\prime-}}-1\right) \int_{\Omega}\left(\beta\left(\omega_{1}\right)-\beta\left(\omega_{2}\right)\right)\left(\omega_{1}-\omega_{2}\right) d x \\
& \quad+\frac{\tau^{p^{+}} C_{M}^{p^{+} / p^{\prime-}}}{p^{-}} \int_{\Omega}\left|\omega_{1}-\omega_{2}\right|^{p(x)} d x .
\end{aligned}
$$

Thus, from Lemma 4 we get

$$
\begin{aligned}
& \tau\left(\frac{1}{2}\right)^{p^{-}} \rho_{p(x)}\left(\nabla\left(\omega_{1}-\omega_{2}\right)\right) \\
& \quad \leq \frac{\tau^{p^{+}} C_{M}^{p^{+} / p^{\prime-}}}{p^{-}} \rho_{p(x)}\left(\nabla\left(\omega_{1}-\omega_{2}\right)\right) .
\end{aligned}
$$

Hence, when $0<\tau<\left((1 / 2)^{p^{-}} p^{-} / C \cdot C_{M}^{p^{+} / p^{\prime-}}\right)^{1 /\left(p^{+}-1\right)}$ we have $\omega_{1}=\omega_{2}$.

\subsection{Case 2: $u_{0} \in L^{2}(\Omega)$}

Theorem 11. Assume that $\left(H_{1}\right),\left(H_{2}\right)$, and $\left(H_{3}\right)$ hold true. Then, for $n=0, \ldots, N$, there exists a unique solution $U^{n}$ of (14) in $W_{0}^{1, p(x)}(\Omega)$ provided that $0<\tau<1 / C$ where $C$ is some positive constant.

Proof. The proofs of existence and uniqueness are the same as those of Theorem 6 . Therefore, we omit them.

Now, we consider the following assumption:

$\left(H_{5}\right)$ for any $\xi \in \mathbb{R}$, the map $(x, t) \longmapsto f(x, t, \xi)$ is measurable and, a.e. in $\Omega \times \mathbb{R}^{+}, \xi \longmapsto f(x, t, \xi)$ is continuous. Furthermore, we assume that there exist $r \in C_{+}(\Omega)$ with $r(x)>\sup (2, p(x))$ and positive constants $C_{5}$ and $C_{6}$ such that

$$
\operatorname{sign}(\xi) f(x, t, \xi) \geq C_{5}|\xi|^{r(x)-1}-C_{6} .
$$

Then, we have the following stability theorem.

Theorem 12. Assume that $\left(H_{1}\right)$ and $\left(H_{5}\right)$ are fulfilled. Then, there exists a constant $C\left(T, u_{0}\right)>0$ such that, for all $n=$ $1, . ., N$,

$$
\begin{aligned}
& \int_{\Omega} \psi^{*}\left(\beta\left(U^{n}\right)\right) d x+\tau \sum_{k=1}^{n}\left\|U^{k}\right\|_{1, p(x)}^{\alpha}+C \tau \sum_{k=1}^{n}\left\|U^{k}\right\|_{q(x)}^{\alpha^{\prime}} \\
& \leq C\left(T, u_{0}\right) \\
& \max _{1 \leq k \leq n} \sum_{k=1}^{n}\left\|\beta\left(U^{k}\right)\right\|_{2}^{2}+\left\|\beta\left(U^{k}\right)-\beta\left(U^{k-1}\right)\right\|_{2}^{2} \\
& \leq C\left(T, u_{0}\right)
\end{aligned}
$$

where $\alpha$ and $\alpha^{\prime}$ are two constants each depending either on $p^{+}$ or on $p^{-}$.
Proof. Since the proof is nearly the same as that of Theorem 7 , we just sketch it.

The argument that allowed us to get (34), with $\left(H_{5}\right)$, allows also us to write

$$
\begin{aligned}
\int_{\Omega} \psi^{*}\left(\beta\left(U^{n}\right)\right) d x+\tau \sum_{k=1}^{N} \rho_{p(x)}\left(\nabla U^{k}\right) \\
\quad+\tau \sum_{k=1}^{N} \rho_{r(x)}\left(U^{k}\right) \\
\leq \tau C_{6} \sum_{k=1}^{N}\left\|U^{k}\right\|_{L^{1}(\Omega)}+\int_{\Omega} \psi^{*}\left(\beta\left(u_{0}\right)\right) d x,
\end{aligned}
$$

By using Lemmas 4 and 5, $\left(H_{1}\right)$ and Young's inequality, we get that for all $\eta>0$ there exists $C_{\eta}\left(T, u_{0}\right)>0$ such that

$$
\begin{gathered}
\int_{\Omega} \psi^{*}\left(\beta\left(U^{n}\right)\right) d x+\tau \sum_{k=1}^{n} \rho_{p(x)}\left(\nabla U^{k}\right) \\
+\tau \sum_{k=1}^{n} \rho_{r(x)}\left(U^{k}\right) \\
\leq \eta \tau \sum_{k=1}^{N} \rho_{p(x)}\left(\nabla U^{k}\right)+C_{\eta}\left(T, u_{0}\right) .
\end{gathered}
$$

Since $\psi^{*}(\beta(u))$ is positive then, for a suitable choice of $\eta$, we infer from (66) that

$$
\tau \sum_{k=1}^{n} \rho_{p(x)}\left(U^{k}\right) \leq \widetilde{C}_{\eta}\left(T, u_{0}\right) .
$$

By taking $\alpha$ and $\alpha^{\prime}$ such that

$$
\begin{gathered}
\left\|U^{k}\right\|_{1, p(x)}^{\alpha}=\min \left(\left\|U^{k}\right\|_{1, p(x)}^{p^{+}},\left\|U^{k}\right\|_{1, p(x)}^{p^{-}}\right), \\
\left\|U^{k}\right\|_{r(x)}^{\alpha^{\prime}}=\min \left(\left\|U^{k}\right\|_{r(x)}^{r^{+}},\left\|U^{k}\right\|_{r(x)}^{q^{-}}\right)
\end{gathered}
$$

and using (66) and (67), we deduce that

$$
\begin{aligned}
& \int_{\Omega} \psi^{*}\left(\beta\left(U^{n}\right)\right) d x+\tau \sum_{k=1}^{n}\left\|U^{k}\right\|_{1, p(x)}^{\alpha}+C \tau \sum_{k=1}^{n}\left\|U^{k}\right\|_{r(x)}^{\alpha^{\prime}} \\
& \quad \leq C\left(T, u_{0}\right) .
\end{aligned}
$$

As in (39), by using $\left(H_{5}\right)$, we get

$$
\begin{aligned}
& \left\|\beta\left(U^{n}\right)\right\|_{L^{2}(\Omega)}^{2}+\sum_{k=1}^{n}\left\|\beta\left(U^{k}\right)-\beta\left(U^{k-1}\right)\right\|_{L^{2}(\Omega)}^{2} \\
& \leq C_{1} \tau\left\|\beta\left(U^{k}\right)\right\|_{L^{1}(\Omega)}+\left\|\beta\left(u_{0}\right)\right\|_{L^{2}(\Omega)}^{2} .
\end{aligned}
$$

Hence, by $\left(H_{1}\right),(67)$, and Lemma 5, we deduce (64). 


\section{Absorbing Sets in $W_{0}^{1, p(x)}(\Omega)$ : Existence of the Attractor}

In this section we consider the following problems: for all integer $n>0$

$$
\begin{array}{r}
\beta\left(U^{n}\right)-\tau \Delta_{p(x)} U^{n}+\tau f\left(x, n \tau, U^{n}\right)=\beta\left(U^{n-1}\right) \\
U^{n}=0 \text { in } \partial \Omega,
\end{array}
$$$$
\text { in } \Omega, \quad(71)
$$

with $U^{0}=u_{0}$ and $\tau$ fixed such that $0<\tau<\tau_{2}$ where $\tau_{2}=$ $\min \left(1,1 / C_{2}\right)$.

We assume that $\left(H_{1}\right),\left(H_{2}\right)$, and $\left(H_{3}\right)$ hold true in all the remaining section.

The result of Theorem 6 on the existence and uniqueness of the solution of (14) allows us to define a map $S_{\tau}$ on $L^{\infty}(\Omega) \cap$ $W^{1, p(x)}(\Omega)$ by setting

$$
S_{\tau} U^{n-1}=U^{n}
$$

Since $S_{\tau}$ is continuous, we have

$$
S_{\tau}^{n} U^{0}=U^{n}
$$

The existence of an absorbing set in $W_{0}^{1, p(x)}(\Omega) \cap L^{\infty}(\Omega)$ allows us to prove the existence of a global compact attractor (see [9]). This will be done next in Theorem 14.

Proposition 13. If $\tau$ satisfies $\tau<1 / C_{2}$ then there is an absorbing set $\mathfrak{B}$ in $W_{0}^{1, p(x)}(\Omega) \cap L^{\infty}(\Omega)$. Namely, for any $u_{0} \in$ $L^{\infty}(\Omega)$ there exists $n(\tau)$ such that

$$
\left\|U^{n}\right\|_{L^{\infty}(\Omega)}+\left\|U^{n}\right\|_{1, p(x)} \leq C, \quad \forall n \geq n(\tau) .
$$

Proof. We multiply (14) by $\Delta_{n}=\left(U^{n}-U^{n-1}\right)$. We obtain

$$
\begin{gathered}
\left\langle\frac{\beta\left(U^{n}\right)-\beta\left(U^{n-1}\right)}{\tau}, U^{n}-U^{n-1}\right\rangle+\left\langle\nabla U^{n}, \nabla \Delta_{n}\right\rangle \\
+\int_{\Omega} f\left(x, n \tau, U^{n}\right) \Delta_{n} d x=0 .
\end{gathered}
$$

Let us denote

$$
F_{\beta}(u)=\int_{0}^{u}\left(f(x, n \tau, \omega)+C_{2} \beta(\omega)\right) .
$$

By $\left(H_{3}\right), F_{\beta}(u)$ is a convex function and hence satisfies the standard inequality

$$
F_{\beta}^{\prime}(u)(u-v) \geq F_{\beta}(u)-F_{\beta}(v),
$$

Consequently,

$$
\begin{aligned}
\left\langle f\left(x, n \tau, U^{n}\right), \Delta_{n}\right\rangle & \\
= & \left\langle f\left(x, n \tau, U^{n}\right)+C_{2} \beta\left(U^{n}\right), \Delta_{n}\right\rangle \\
& -C_{2}\left\langle\beta\left(U^{n}\right), \Delta_{n}\right\rangle \\
\geq & \int_{\Omega}\left(F_{\beta}\left(U^{n}\right)-F_{\beta}\left(U^{n-1}\right)\right) d x \\
& -C_{2}\left\langle\beta\left(U^{n}\right), \Delta_{n}\right\rangle .
\end{aligned}
$$

Now, by using $\left(H_{1}\right)$, we get that $\psi(u)$ is a convex function and hence we have

$$
\psi^{\prime}(v)(u-v) \geq \psi(u)-\psi(v) .
$$

Thus, we obtain

$$
\begin{aligned}
\int_{\Omega} & \beta\left(U^{n}\right)\left(U^{n}-U^{n-1}\right) d x \\
= & \int_{\Omega}\left(\beta\left(U^{n}\right)-\beta\left(U^{n-1}\right)\right)\left(U^{n}-U^{n-1}\right) d x \\
& +\int_{\Omega} \beta\left(U^{n-1}\right)\left(U^{n}-U^{n-1}\right) d x \\
\leq & \int_{\Omega}\left(\beta\left(U^{n}\right)-\beta\left(U^{n-1}\right)\right)\left(U^{n}-U^{n-1}\right) d x \\
& +\int_{\Omega}\left(\psi\left(U^{n}\right)-\psi\left(U^{n-1}\right)\right) d x .
\end{aligned}
$$

The following inequality holds, for any $a$ and $b$ in $\mathbb{R}^{N}$ :

$$
\frac{1}{p^{+}}|a|^{p(x)}-\frac{1}{p^{-}}|b|^{p(x)} \leq|a|^{p(x)-2} a \cdot(a-b),
$$

By setting $a=\nabla U^{n}$ and $b=\nabla U^{n-1}$ and integrating over $\Omega$, we get

$$
\begin{aligned}
& \left(\frac{1}{p^{+}} \rho_{p(x)}\left(U^{n}\right)-\frac{1}{p^{-}} \rho_{p(x)}\left(U^{n-1}\right)\right) \\
& \quad \leq \int_{\Omega}\left|\nabla U^{n}\right|^{p(x)-2} \nabla U^{n}\left(\nabla U^{n}-\nabla U^{n-1}\right) .
\end{aligned}
$$

Now, since $\tau<1 / C_{2}$, then from (75), we deduce that

$$
\begin{aligned}
& \frac{1}{p^{+}} \rho_{p(x)}\left(\nabla U^{n}\right)+\int_{\Omega} F_{\beta}\left(U^{n}\right) d x \\
& \leq \frac{1}{p^{-}} \rho_{p(x)}\left(\nabla U^{n-1}\right)+\int_{\Omega} F_{\beta}\left(U^{n-1}\right) d x \\
& \quad+C_{2} \int_{\Omega}\left(\psi\left(U^{n}\right)-\psi\left(U^{n-1}\right)\right) d x
\end{aligned}
$$

On the other hand, by writing

$$
\int_{\Omega} F_{\beta}(u) d x=\int_{\Omega} F(u) d x+C_{2} \int_{\Omega} \psi(u) d x,
$$

where $F(u)=\int_{0}^{u} f(x, t, \omega) d \omega$, we have

$$
\begin{aligned}
& \frac{1}{p^{+}} \rho_{p(x)}\left(\nabla U^{n}\right)+\int_{\Omega} F\left(U^{n}\right) d x \\
& \quad \leq \frac{1}{p^{-}} \rho_{p(x)}\left(\nabla U^{n-1}\right)+\int_{\Omega} F\left(U^{n-1}\right) d x .
\end{aligned}
$$

Denote the left hand side of $(85)$ by $y^{n}$. By using $\left(H_{1}\right)$ and relations (23) and (24) of Theorem 7 and taking $N \tau=1$, we deduce that there exists $n_{\tau}$ such that

$$
\tau \sum_{n=n_{0}}^{n_{0}+N} y^{n} \leq a_{1} \quad \text { for all } n_{0} \geq n_{\tau}
$$


Then, by applying the discrete version of the uniform Gronwall Lemma (see Lemma 7.5 of [11]) with $h_{n}=0$, we obtain

$$
\frac{1}{p^{+}} \rho_{p(x)}\left(\nabla U^{n}\right)+\int_{\Omega} F\left(U^{n}\right) d x \leq C \quad \text { for all } n \geq n_{\tau} \text {. }
$$

Thus by Lemma 5, we deduce that

$$
\left\|U^{n}\right\|_{1, p(x)} \leq C \quad \text { for all } n \geq n_{\tau}
$$

Therefore, from (88) and Theorem 7, we conclude to the desired relation

$$
\left\|U^{n}\right\|_{L^{\infty}(\Omega)}+\left\|U^{n}\right\|_{1, p(x)} \leq C, \quad \forall n \geq n(\tau) .
$$

Now we are able to state our result on the existence of a compact attractor.

Theorem 14. Suppose that $f(x, t, \xi)=f(x, \xi)$. Then, for $u_{0} \in$ $L^{\infty}(\Omega)$, the discretized problem (71) has an associated semigroup solution $S_{\tau}$ that maps $L^{\infty}(\Omega)$ into $L^{\infty}(\Omega) \cap W_{0}^{1, p(x)}(\Omega)$. This semigroup has a compact attractor $\mathfrak{A}_{\tau}$ which is bounded in $L^{\infty}(\Omega) \cap W_{0}^{1, p(x)}(\Omega)$.

Proof. The nonlinear map $S_{\tau}$ defines a semigroup from $L^{\infty}(\Omega)$ into $L^{\infty}(\Omega) \cap W_{0}^{1, p(x)}(\Omega)$. By Proposition 13, the existence of an absorbing ball $\mathfrak{B}_{\tau}$ in $L^{\infty}(\Omega) \cap W_{0}^{1, p(x)}(\Omega)$ is guaranteed.

We define the $\omega$ - limit set of $\mathfrak{B}_{\tau}$ as

$$
\mathfrak{A}_{\tau}=\omega\left(\mathfrak{B}_{\tau}\right)=\bigcap_{n \geq 0} \overline{\bigcup_{m \geq n} S_{\tau}^{m}\left(\mathfrak{B}_{\tau}\right)}
$$

Then, by the results of Temam (see [9]), $\mathfrak{A}_{\tau}=\omega\left(\mathfrak{B}_{\tau}\right)$ is a compact attractor which attracts all bounded sets of $L^{\infty}(\Omega)$, which means that, for all $u_{0} \in L^{\infty}(\Omega)$, we have

$$
\operatorname{dist}\left(\mathfrak{\mathcal { U }}_{\tau}, S_{\tau}^{n} u_{0}\right) \longmapsto 0 \text { as } n \longrightarrow+\infty \text {. }
$$

\section{Data Availability}

The authors declare that no data were used to support this study.

\section{Conflicts of Interest}

The authors declare that they have no conflicts of interest.

\section{References}

[1] G. Akagi, "Doubly nonlinear parabolic equations involving variable exponents," Discrete and Continuous Dynamical Systems - Series S, vol. 7, no. 1, pp. 1-16, 2014.

[2] S. N. Antontsev, J. I. Daz, and S. Shmarev, "Energy methods for free boundary problems. Applications to nonlinear PDEs and fluid mechanics," in Progress in Nonlinear Differential Equations and their Applications, vol. 48, Birkhäuser, Boston, 2002.
[3] S. N. Antontsev and S. I. Shmarev, "A model porous medium equation with variable exponent of nonlinearity: existence, uniqueness and localization properties of solutions," Nonlinear Analysis: Theory, Methods \& Applications, vol. 60, no. 3, pp. 515545, 2005.

[4] S. Antontsev and S. Shmarev, "Existence and uniqueness for doubly nonlinear parabolic equations with nonstandard growth conditions," Differential Equations \& Applications, vol. 4, no. 1, pp. 67-94, 2012.

[5] S. Antontsev and S. Shmarev, "Doubly degenerate parabolic equations with variable nonlinearity I: existence of bounded strong solutions," Advances in Differential Equations, vol. 17, no. 11-12, pp. 1181-1212, 2012.

[6] S. N. Antontsev and S. Shmarev, "Evolution PDEs with nonstandard growth conditions. Existence, uniqueness, localization, blow-up," in Atlantis Studies in Differential Equations, vol. 4, pp. 369-391, Atlantis Press, Paris, 2015.

[7] A. EL Hachimi and H. El Ouardi, "Existence and regularity of a global attractor for doubly nonlinear parabolic equations," Electronic Journal of Differential Equations, vol. 2002, no. 45, pp. $1-15,2002$.

[8] D. Stan and J. L. Vázquez, "Asymptotic behavior of the doubly nonlinear diffusion equation ut $=\Delta$ pum on bounded domains," Nonlinear Analysis: Theory, Methods \& Applications, vol. 77, pp. 1-32, 2013.

[9] R. Temam, Infinite Dimensional Dynamical Systems in Mechanics and Physics, vol. 68 of Applied Mathematical Sciences, Springer-Verlag, New York, NY, USA, 1988.

[10] F. Benzekri and A. El Hachimi, "Doubly nonlinear parabolic equations related to the p-Laplacian operator: semidiscretization," Electronic Journal of Differential Equations, vol. 2003, no. 113, pp. 1-14, 2003.

[11] A. Eden, B. Michaux, and J. M. Rakotoson, "Semi-discretized nonlinear evolution equations as dynamical systems and error analysis," Indiana University Mathematics Journal, vol. 39, no. 3, pp. 737-783, 1990.

[12] Z. Chaouai and A. El Hachimi, "Qualitative properties of nonnegative solutions for a doubly nonlinear problem with variable exponents," Abstract and Applied Analysis, vol. 2018, Article ID 3821217, 14 pages, 2018.

[13] M. Ružička, Electrorheological Fluids: Modeling and Mathematical Theory, vol. 1748 of Lecture Notes in Mathematics, SpringerVerlag, Berlin, Germany, 2000.

[14] L. Diening, P. Harjulehto, P. Hasto, and M. Ruzicka, "Lebesgue and Sobolev spaces with variable exponents," in Lecture Notes in Mathematics, vol. 2017, Springer-Verlag, Heidelberg, 2011.

[15] X. L. Fan and D. Zhao, "On the spaces $\operatorname{Lp}(\mathrm{x})(\Omega)$ and Wm, $\mathrm{p}(\mathrm{x})(\Omega)$," Journal of Mathematical Analysis and Applications, vol. 263, no. 2, pp. 424-446, 2001.

[16] X. L. Fan, J. S. Shen, and D. Zhao, "Sobolev embedding theorems for spaces $W^{k, p(x)}(\Omega)$," Journal of Mathematical Analysis and Applications, vol. 262, no. 2, pp. 749-760, 2001.

[17] J. Musielak, Orlicz Spaces and Modular Spaces, vol. 1034 of Lecture Notes in Mathematics, Springer-Verlag, Berlin, Germany, 1983.

[18] Y. Fu, "The existence of solutions for elliptic systems with nonuniform growth," Studia Mathematica, vol. 151, no. 3, pp. 227-246, 2002.

[19] X.-L. Fan and Q.-H. Zhang, "Existence for $\mathrm{p}(\mathrm{x})$-Laplacien Dirichlet problem," Nonlinear Analysis, vol. 52, no. 8, pp. 18431852, 2003. 


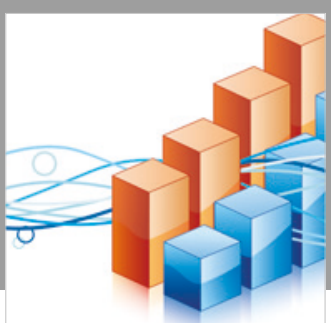

Advances in

Operations Research

\section{-n-m}
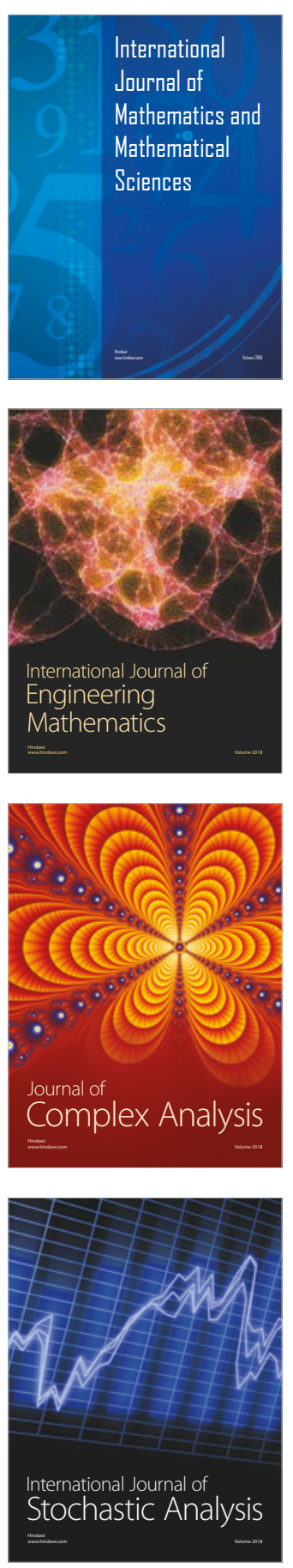
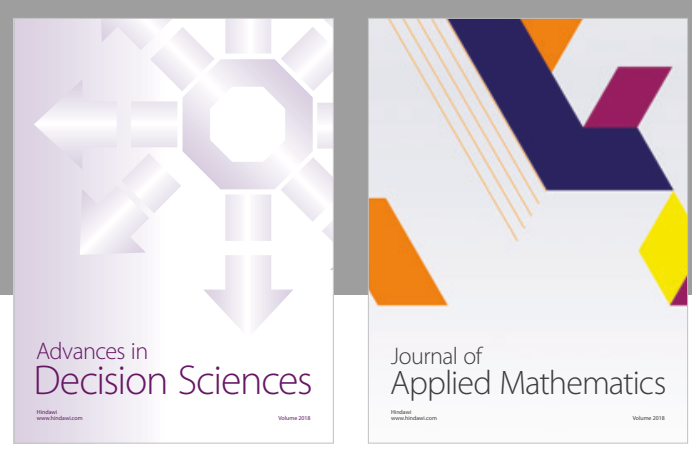

Journal of

Applied Mathematics
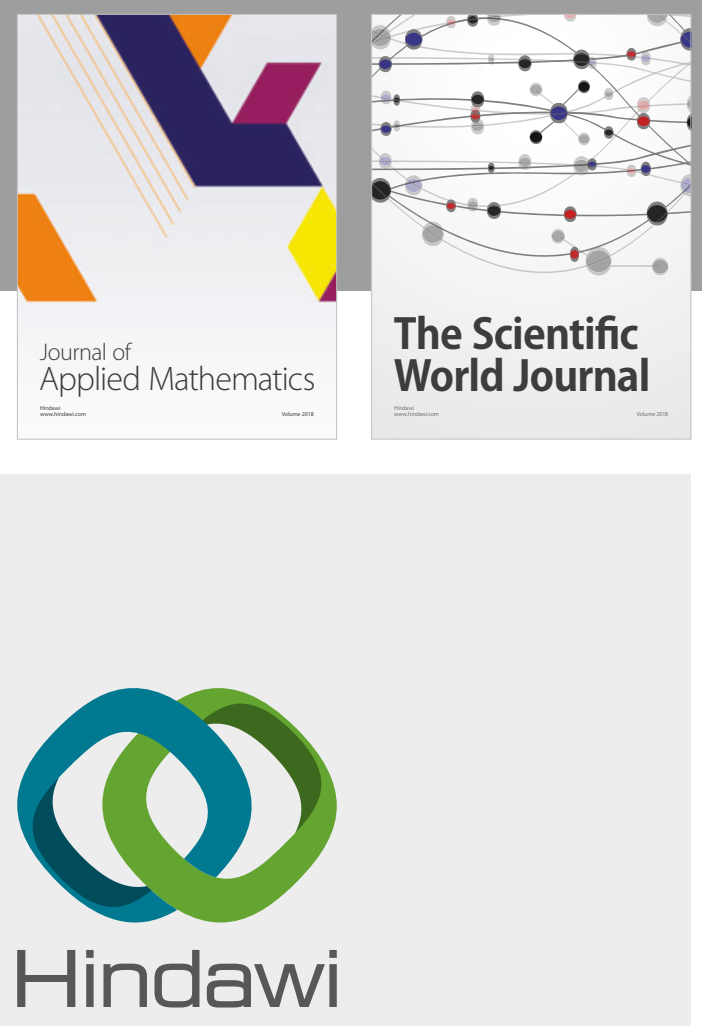

Submit your manuscripts at

www.hindawi.com

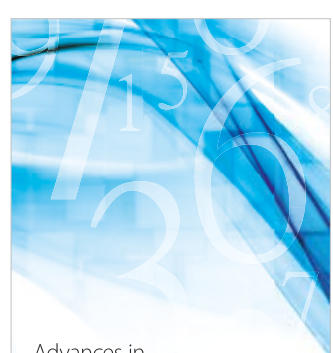

Advances in
Numerical Analysis
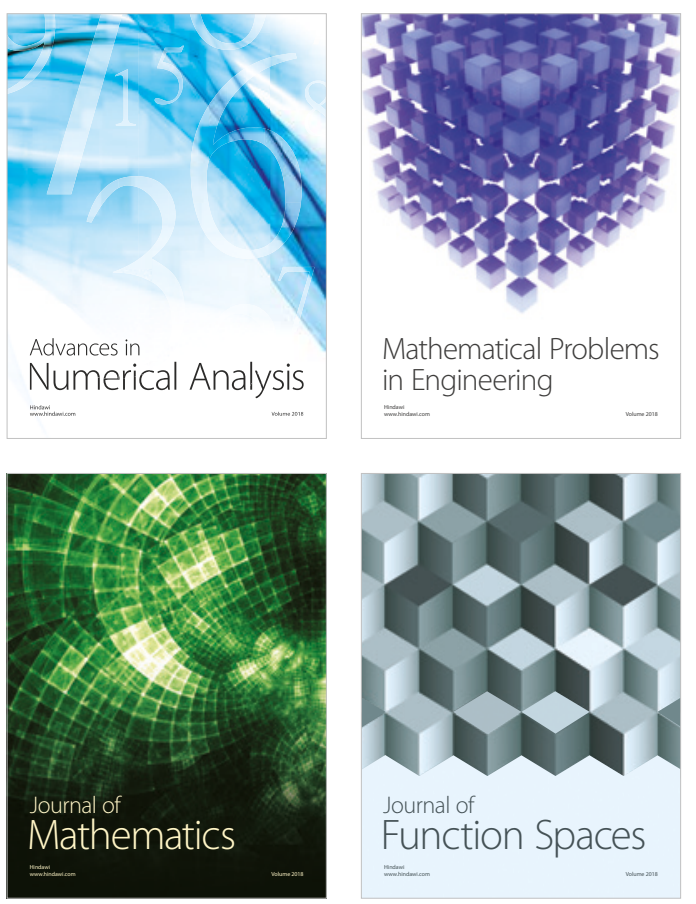

Mathematical Problems in Engineering

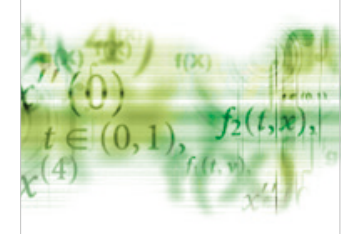

International Journal of

Differential Equations

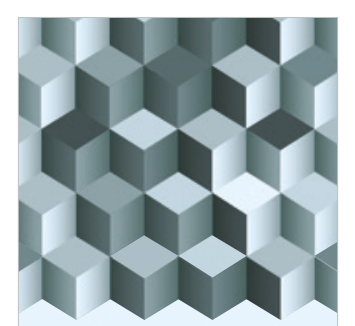

Journal of

Function Spaces

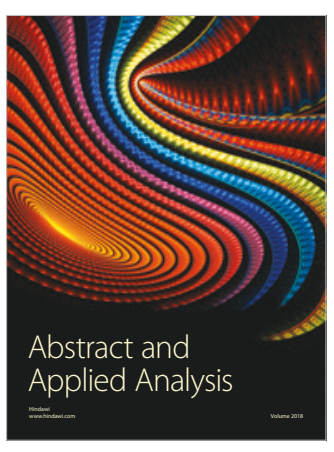

The Scientific

World Journal

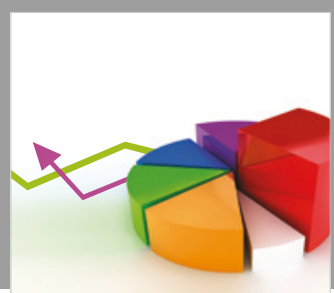

Journal of

Probability and Statistics
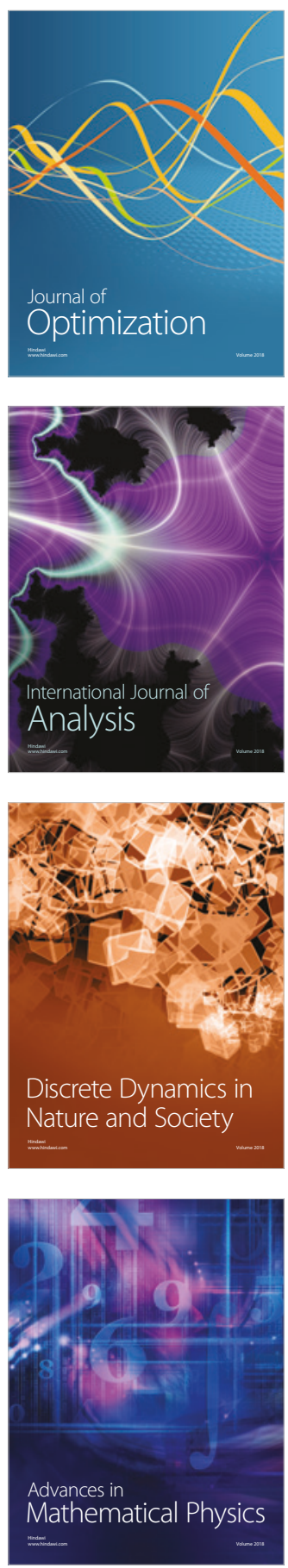